\title{
A transient cutaneous relapse of AML M1 in hematological remission: a case report
}



\begin{abstract}
Leukemia cutis (LC) is described as cutaneous infiltration by neoplastic leukocytes into the epidermidis, dermis, or subcutis, resulting in clinically various skin lesions. When the infiltrate is characterized by neoplastic granulocytic precursors, LC is defined as granulocytic sarcoma. Multiple, erythematous, and infiltrated papules and nodules localized on the legs, arms, and trunk are the most common clinical presentation. Here we report a case of granulocytic sarcoma in a patient with a previous diagnosis of acute myeloid leukemia currently in hematological remission.
\end{abstract}

Keywords: leukemia cutis, granulocytic sarcoma, acute myeloid leukemia

Received: 21 April 2017 | Returned for modification: 26 June 2017 | Accepted: 9 July 2017

\section{Introduction}

Leukemia cutis (LC) is described as cutaneous infiltration by neoplastic leukocytes into the epidermidis, dermis, or subcutis, resulting in clinically various skin lesions. When the infiltrate is characterized by neoplastic granulocytic precursors, LC is defined as granulocytic sarcoma or myeloid sarcoma (1). We present a case of granulocytic sarcoma in a patient with a previous diagnosis of acute myeloid leukemia (AML) currently in hematological remission.

\section{Case report}

A 76-year-old woman was admitted to our dermatology department because of the onset of a non-pruritic erythematous papulonodular rash mainly involving the trunk (Figs. 1-2). The patient had no fever, and physical examination was negative for lymphadenopathies and hepatosplenomegaly.

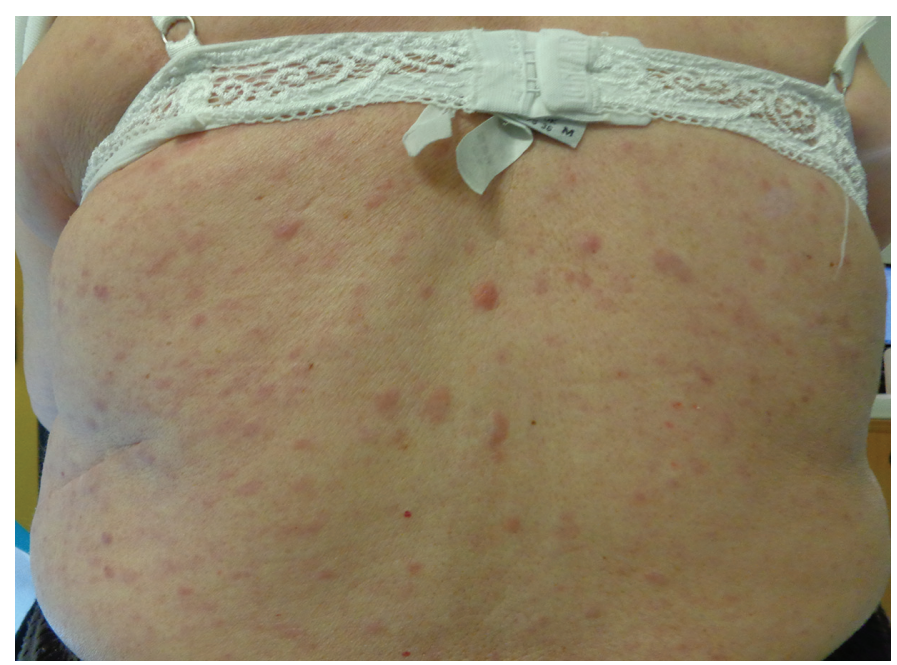

Figure 1 | Erythematous papulo-nodular rash of the trunk.

Two years earlier, she had been diagnosed with AML M1 according to the FAB classification and she was treated with a chemotherapy regimen of cytarabine at intermediate doses for 1 year with a complete remission of the disease. At that time, immunophenotypic analysis on peripheral blood and bone marrow aspirate revealed the presence of a cluster of myeloid blasts positive for $\mathrm{CD}_{33}+, \mathrm{CD}_{34+}$, $\mathrm{CD} 13+$, and $\mathrm{CD}_{117+}$.

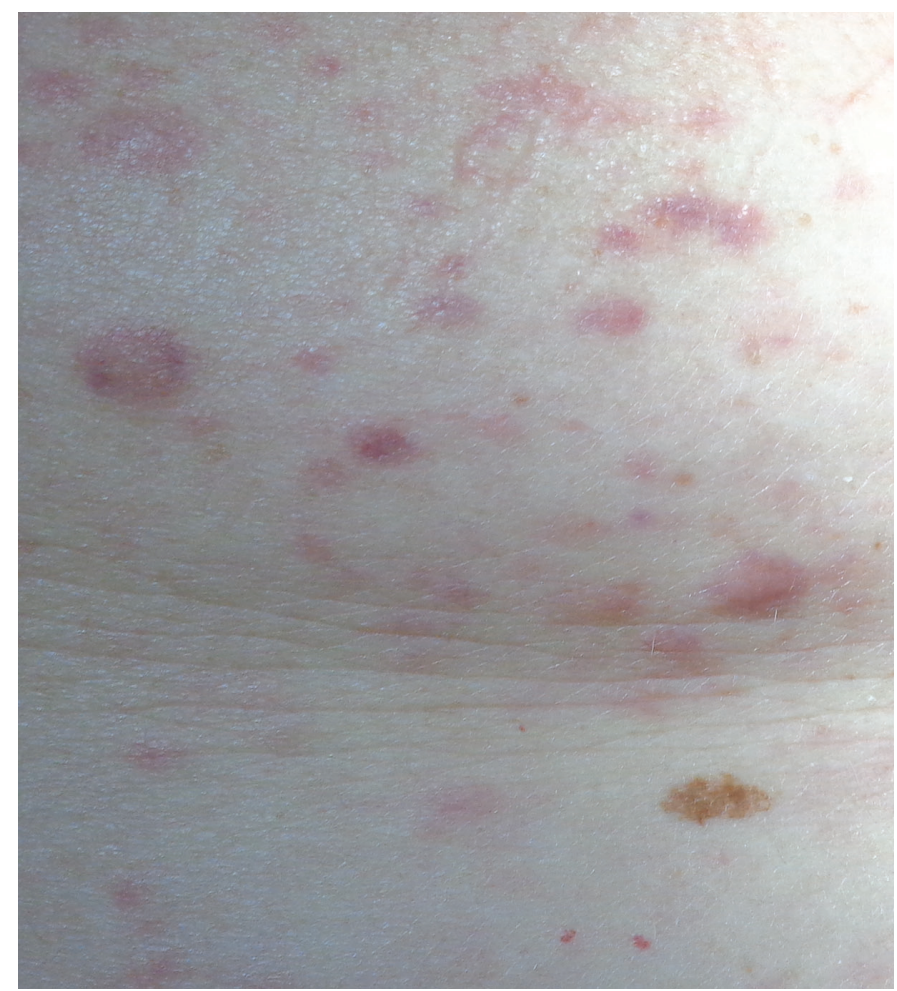

Figure 2 | Close-up of the erythematous papulo-nodular lesion.

Three months before admission to our department, her laboratory parameters were as follows: leukocytes $4,120 / \mathrm{mm}^{3}$, neutrophils $48 \%$, monocytes $5 \%$, hemoglobin $11.9 \mathrm{~g} / \mathrm{dl}$, platelets $139,000 / \mathrm{mm}^{3}$, normal LDH, myelocytes $2 \%$, and metamyelocytes $4 \%$ with the appearance of $1 \%$ blasts. Because of this detection on peripheral blood, the hematologist performed a bone marrow aspirate, which was hypercellular $\left(111,000 / \mathrm{mm}^{3}\right)$ with $1 \%$ blasts positive for $\mathrm{CD}_{33}+, \mathrm{CD}_{34}+$, and $\mathrm{CD} 117^{+}$(Figs. 3-4). These results confirmed complete remission of the disease. 


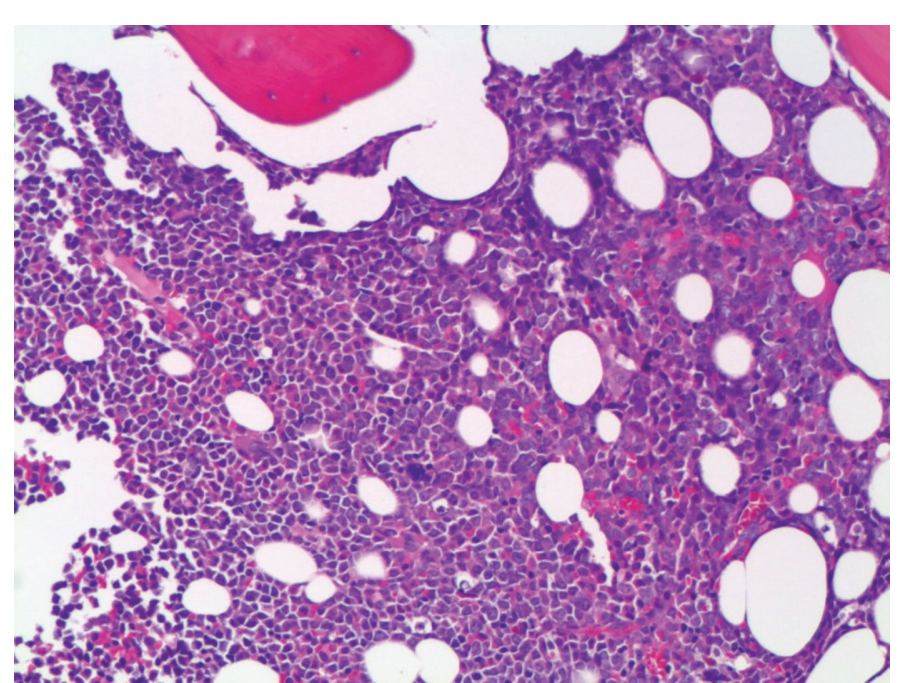

Figure 3 | Bone marrow. Histopathological findings (H\&E, 100x).

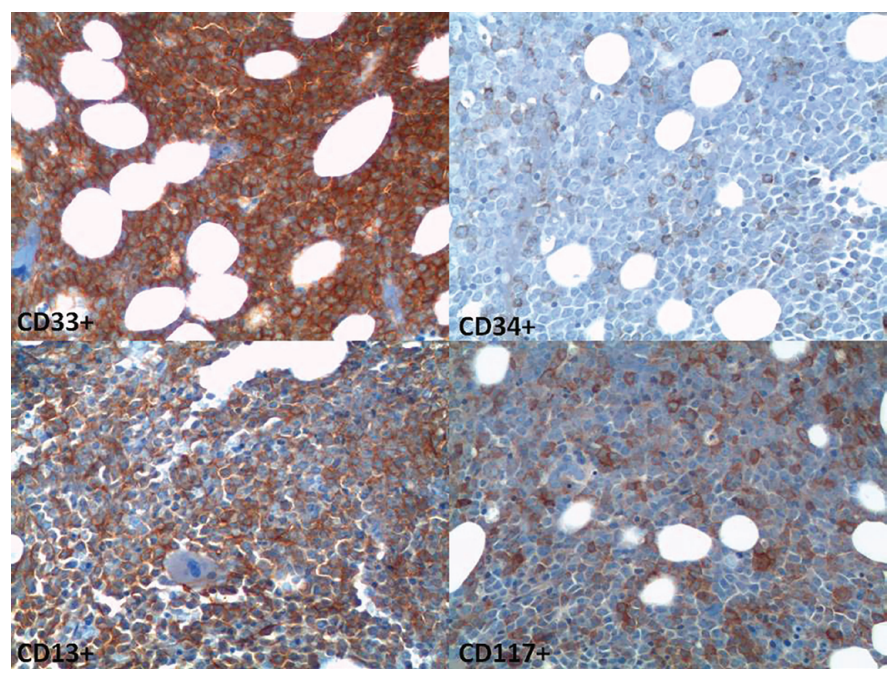

Figure 4 | Bone marrow. Immunohistochemical features: positivity for CD33+, CD34+, CD13+, CD117+ (200x).

The patient was otherwise in good health, and so there was no need for hospitalization. However, a punch biopsy was performed on the skin eruption. We examined the patient once a week for a month, waiting for the results of the histological examination.

The histological assessment showed a periadnexal and perineural neoplastic infiltrate with mitotic figures and sclerosis with positivity of the cells for $\mathrm{CD}_{33}+, \mathrm{CD}_{34}+$, $\mathrm{CD}_{13}+$, and $\mathrm{CD} 117+$ on immunohistochemical staining, which were findings compatible with a diagnosis of granulocytic sarcoma (Figs. 5-6). This infiltrate had the same immunophenotype of the blasts found 2 years earlier at the onset of the AML. Laboratory tests had parameters on peripheral blood and bone marrow aspirate that were the same of those encountered 3 months earlier with the presence of $1 \%$ blasts positive for $\mathrm{CD}_{33}+$, $\mathrm{CD}_{34+}$, and $\mathrm{CD}_{117+}$, confirming complete remission of AML.

During the month of follow-up, the skin eruption disappeared without any therapy. This atypical occurrence was defined as a transient cutaneous relapse of AML M1 in hematological remission. The patient will continue follow-up with detailed checkups for the risk of relapse of AML M1.

\section{Discussion}

LC comprises a wide range of cutaneous manifestations of any type of leukemia because it has been described in patients with

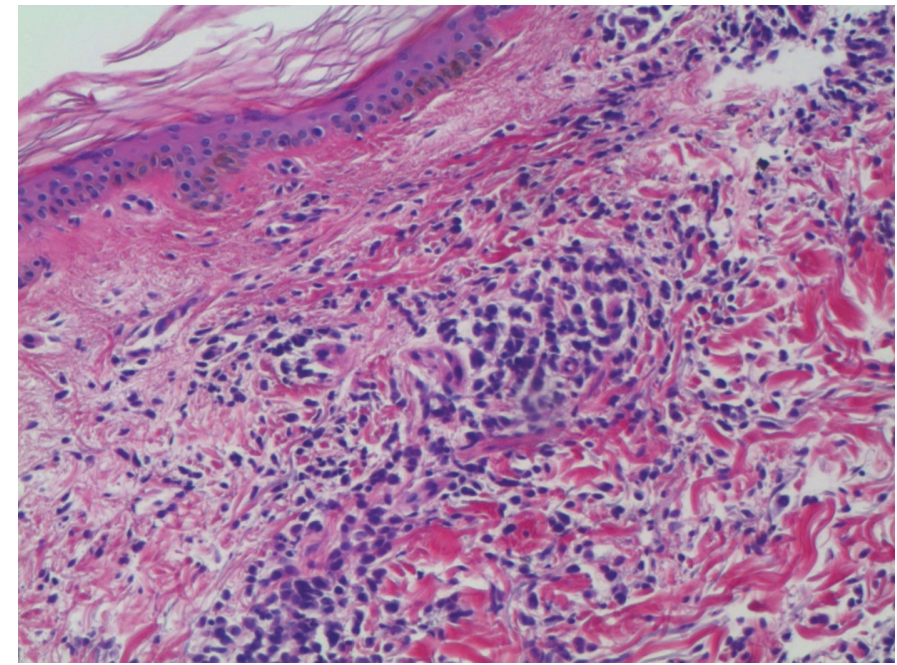

Figure 5 | Skin. Histopathological findings (H\&E, 100x).

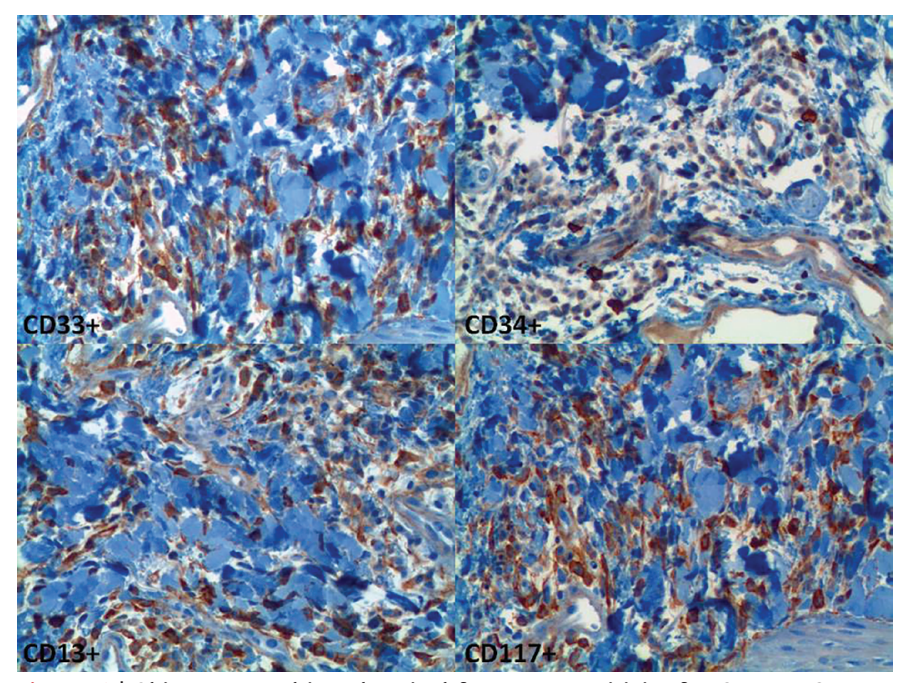

Figure 6 | Skin. Immunohistochemical features: positivity for CD33+, CD34+, CD13+, CD117+ (200x).

AML, chronic myeloproliferative diseases, including chronic myelogenous leukemia, myelodysplastic syndromes, and myelodysplastic/lymphoproliferative diseases. LC is more frequent in patients with AML, and in particular it can be found in myelomonocytic (FAB-M4) and monocytic (FAB-M5) subtypes (1-2).

The majority of cases of LC have been observed after a diagnosis of systemic leukemia; occasionally (<10\% of cases) skin infiltration can occur before bone marrow or peripheral blood involvement. Aleukemic leukemia cutis (ALC) is the term for this uncommon event, in which skin invasion by leukemic blasts occurs at least 1 month prior to the appearance of the disease in bone marrow or peripheral blood (3-4).

LC can be defined as myeloid sarcoma or granulocytic sarcoma, in which the infiltrate is characterized by neoplastic granulocytic precursors; otherwise, when the infiltrate is composed of neoplastic monocytic precursors (monoblasts and promonocytes), LC can be labeled monoblastic sarcoma (1).

LC is more frequent in children than in adults. Multiple, erythematous, and infiltrated papules and nodules of various sizes are the most common clinical presentation of LC; skin lesions are localized most commonly on the legs, arms, and trunk (1-2).

Skin biopsy is crucial for the diagnosis of LC because only histological features, and most importantly the immunophenotypic pattern, allow identification of the disease. The histological examination of granulocytic sarcoma, the subtype of LC composed 
of neoplastic granulocytic precursors, shows a perivascular and periadnexal distribution of the infiltrate, with stromal fibrosis; neoplastic cells are usually large, with abundant eosinophilic cytoplasm and large nuclei with finely dispersed chromatin and occasional small nucleoli. Positivity for MPO, CD34+, and CD117+ on immunohistochemical staining confirms the diagnosis of granulocytic sarcoma (1-5).

In our case, neoplastic cells of the skin infiltrate were positive for $\mathrm{CD}_{33}+$, $\mathrm{CD}_{34+}$, $\mathrm{CD}_{13}+$, and $\mathrm{CD}_{117+}$, and this immunophenotypic pattern was the same as that found 2 years earlier in peripheral blood and bone marrow. This resulted in a diagnosis of AML M1, treated for 1 year with chemotherapy with complete remission of the disease.

These histopathological findings were supported by laboratory tests on peripheral blood and bone marrow because of the high risk of relapse of systemic leukemia. In the end, laboratory data

\section{References}

1. Cho-Vega JH, Medeiros LJ, Prieto VG, Vega F. Leukemia cutis. Am J Clin Pathol. 2008;129:130-42.

2. Hahn WC, Jones D, Leavitt P, Garber J, Stone R, Skarin AT. Diagnosis in oncology. Leukemia cutis. J Clin Oncol. 1997;15:2170-1.

3. Yonal I, Hindilerden F, Coskun R, Dogan OI, Nalcaci M. Aleukemic leukemia cutis manifesting with disseminated nodular eruptions and a plaque preceding acute monocytic leukemia: a case report. 2011;4:547-54. confirmed complete remission of AML, allowing the diagnosis of ALC.

ALC is extremely rare and it is has a poor prognosis. The mean survival time is 3 to 30 months once neoplastic cells appear in the peripheral blood or bone marrow. Because of its variable pattern of clinical presentation, it is misdiagnosed, resulting in early bone marrow relapse and a poor treatment response. Early recognition of the disease should be emphasized because early diagnosis may result in a better prognosis (3-6).

Radiotherapy, chemotherapy, and total body electron therapy are possible treatment options for ALC, but at present there is no therapy of choice because of the rarity of the disease and the lack of randomized controlled studies (3).

In our case, the skin rash disappeared in 1 month without any therapy, but it will be necessary to follow the patient over time due to the concrete risk of relapse of the disease.

4. Husak R, Blume-Peytaki U, Orfanos CE. Aleukemic leukemia cutis in an adolescent boy. N Engl J Med. 1999;340:893-4.

5. di Meo N, Stinco G, Trevisan G. Cutaneous B-cell chronic lymphocytic leukaemia resembling a granulomatous rosacea. Dermatol Online J. 2013;19:20033.

6. Wilkins R, Janes S. Aleukaemic leukaemia cutis: case report and review of the literature. Clin Lab Haematol. 2004;26:73-5. 